deficiency. Maintenance therapy consists of L-carnitine $100 \mathrm{mg} /$ $\mathrm{kg} /$ daily in infants and children. There are no known serious side effects of L-carnitine. (Breningstall GN. Carnitine deficiency syndromes. Pediatr Neurol March $1990 ; 6: 75-81)$.

OOMMENT. Early diagnosis of carnitine deficiency syndromes and prompt supplementation with oral carnitine may reduce mortality since oral carnitine administration in recommended doses is free from adverse effects except for occasional diarrhea. Supplementation with carnitine is recomended in infants and children with acute or recurrent encephalopathies, myelopathies, or cardionyopathies associated with proven or presumed carnitine deficiencies. Meat products, especially red meats and dairy products, are important dietary sources of carnitine which maintain tissue stores.

\title{
NEW TREATMENT FOR PHENYLKETONURIA
}

Sixteen adolescents and young adults with phenylketonuria were treated with a mixture of valine, isoleucine, and leucine for four 3-month periods, and biochemical and neuropsychological tests were carried out before and after treatment at the Metabolic Disease Center, Children's Hospital Medical Center, University of Cincinnati College of Medicine. The performance of timed attentional tests and a continuous performance test was improved during the valine, isoleucine, and leucine periods compared to the control mixture periods. The attention diagnostic method, a test with strong attentional components, showed significant improvements. These results were consistent with earlier reports of improvement in specific cognitive processes with valine, isoleucine, and leucine treatment in patients who were unable to maintain low serum phenylalanine levels. (Berry HK et al. Valine, isoleucine, and leucine. A new treatment for phenylketonuria. AJDC May $1990 ; 144: 539-543)$.

COMMENT. Phenylalanine and other large neutral amino acids share common receptocytes on a blood brain barrier transport system. The administration of other large neutral amino acids to patients with elevated plasma phenylalanine may reduce the amount of phenylalanine reaching the brain and prevent further deficits in cognition. The amino acid mixture consisted of $150 \mathrm{mg} / \mathrm{kg}$ Valine, $150 \mathrm{mg} / \mathrm{kg}$ Isoleucine, and $200 \mathrm{mg} / \mathrm{kg}$ Leucine. This mixture was prescribed as a supplement to the low phenylalanine formula.

\section{SKIN BIOPSY IN GLYOOGENOSIS TYPE III}

Electron microscopy of skin specimens of five patients with glycogenosis type III were correlated with clinical, biochemical, and electrophysiological findings from the Divisions of Neuropathology and Neuropediatrics, Ciudad Sanitaria Valle de Hebron, Barcelona, Spain. The disease began in infancy in four patients and at 38 years of age in one adult patient. Massive glycogen storage was observed in epithelial secretory cells of eccrine sweat glands and other cells, including Schwann cells of myelinated and unmyelinated fibers, were not 
affected. The changes differed clearly from those in glycogenosis II where glycogen storage is membrane bound and is found in most skin cells including fibroblasts, sweat glands, smooth muscle fibers, endothelial cells, and Schwann cells. Skin biopsy appeared to be useful in diagnosis of glycogenosis III and its differentiation from other glycogen storage disorders. (Sancho S et al. Skin biopsy findings in glycogenosis III: Clinical, biochemical, and electrophysiological correlations. Ann Neurol May 1990; 27:480-486).

COMMENT. Glycogen storage disease type III (debrancher deficiency, Cori-Forbes disease) is an autosamal recessively inherited disease with a deficiency in amyl-1,6-glycosidase or debrancher enzyme. Enzyme deficiencies occur in liver, muscle, heart, leukocytes, erythrocytes, fibroblasts, and muscle cultures. Abnormal glycogen storage is found in liver, muscle, and erythrocytes. Clinically there are three types, 1) infantile, 2) childhood, 3) adult type. The infantile type is associated with hypoglycemia, failure to thrive, and hepatomegaly. Symptams usually remit partially with growth. Childhood types present with heart failure and exercise intolerance. Adults develop gradual weakness and wasting of distal muscles and may have a history of abdaminal enlargement with hepatic dysfunction in early childhood. EMG shows diffuse myopathic changes. Growth failure and hepatic dysfunction including hypoglycemia have been improved by the administration of cornstarch (Borowitz SM, Greene HL. J Pediatr Gastroenterol Neutr 1987; 6:631).

\section{TOXIC DISORDERS}

FETAL HYDANTOIN SYNDROME

In a prospective study of 19 pregnancies monitored by amniocentesis at the Center for Human Genetics and the Meyer Rehabilitation Institute, University of Nebraska, Omaha, an adverse outcome was predicted for four fetuses on the basis of low epoxide hydrolase activity ( $30 \%$ of standard). The mothers were receiving phenytoin monotherapy and the infants had clinical characteristics of the fetal hydantoin syndrome. Fifteen fetuses with enzyme activity above $30 \%$ of the standard had no features of the syndrome. The authors suggest that this enzymatic biomarker may be useful in the prediction of infants at increased risk for congenital malformations induced by anticonvulsant drugs. (Buehler BA et al. Prenatal prediction of risk of the fetal hydantoin syndrome. N Engl J Med May 31, 1990; $322: 1567-72$ ).

form oxidative Anticonvulsant drugs that are metabolized to teratogenic risk to the fetus. The measurement of the enzyme involved with the biotransformation of the epoxide to a less toxic metabolite may serve as a biomarker of the fetus at high risk for the fetal hydantoin syndrome. 